\title{
Clinical Characteristics of Vaginal Discharge Associated Streptococcus pyogenes at General Japanese Hospital
}

\author{
Masaaki Minami ${ }^{1 *}$, Ryoko Sakakibara², Mika Watanabe², Hideo Morita², Naoto Kanemaki ${ }^{3}$, \\ Michio Ohta ${ }^{4}$
}

${ }^{1}$ Department of Bacteriology, Graduate School of Medical Sciences, Nagoya City University, Nagoya, Japan

${ }^{2}$ Department of Clinical Investigation, Daido Hospital, Nagoya, Japan

${ }^{3}$ Department of Gastroenterology, Daido Hospital, Nagoya, Japan

${ }^{4}$ School of Nursing, Sugiyama Jyogakuen University, Nagoya, Japan

Email:^minami@med.nagoya-cu.ac.jp

How to cite this paper: Minami, M., Sakakibara, R., Watanabe, M., Morita, H., Kanemaki, N. and Ohta, M. (2018) Clinical Characteristics of Vaginal Discharge Associated Streptococcus pyogenes at General Japanese Hospital. Journal of Biosciences and Medicines, 6, 9-16.

https://doi.org/10.4236/jbm.2018.61002

Received: November 19, 2017 Accepted: December 26, 2017 Published: December 29, 2017

\begin{abstract}
Streptococcus pyogenes ( $S$. pyogenes) is popular pathogen caused various infectious disease. This study was conducted to find out the recent clinical characteristics of $S$. pyogenes isolates from vaginal discharge at Japanese hospital. $S$. pyogenes was identified by standard laboratory procedure. Antimicrobial susceptibility testing was performed by microdilution assay according to CLSI recommendation. Total $61 S$. pyogenes were isolated from vaginal discharge. The major age incidence among 0 - 10 years age group was 14 (23\%), among 21 - 30 years age group, 7 (11.5\%), among 31 - 40 years age group, $13(21.3 \%)$, among 51 - 60 years age group, $8(13.1 \%)$, and among 61 - 70 years age group, $11(18 \%)$. The numbers of ciprofloxacin and clarithromycin no-susceptible $S$. pyogenes from 0 - 10, 31 - 40, and 61-70 years age group were higher than those from other groups. The numbers of clindamycin and minocycline no susceptible $S$. pyogenes from 0 - 10, and 21 - 40 years age group were higher than those from other group. Furthermore, our study revealed that clarithromycin was completely ineffective in $21-30$ years age group $(p<0.05)$. As the numbers of under and not less than 13 year age group were 15, and 46, respectively, the number of adult cases was higher than the number of child cases. Clarithromycin no-susceptible rate was highest and minocycline nosusceptible rate was lowest in both group. There was no significant difference of antimicrobial susceptible trends between under and not less than 13 year age groups. Our study suggests the need for continuous epidemiological surveillance of vaginal discharge associated $S$. pyogenes because the trend of infectious disease epidemiology is always changing drastically.
\end{abstract}




\section{Keywords}

Streptococcus pyogenes, Susceptibility, Antimicrobial Resistance, Vaginal Discharge, Clarithromycin

\section{Introduction}

Bacterial vaginosis is the most prevalent cause of vaginal discharge in reproductive age women [1], is present in $\sim 30 \%$ of women in the United States [2] and is characterized by vaginal colonization with anaerobic bacterial species and a loss of normal lactobacilli. The clinical presentation of Bacterial vaginosis is characterized by an odorous discharge or no symptoms at all, without the redness, swelling or pain typical of inflammation. However, at the mucosal level, this condition has a significant pro-inflammatory impact that is associated with some miserable clinical outcomes [3]. Bacterial vaginosis is known to be common vaginal infection worldwide [4]. It is associated with important public health issues, such as preterm labor and the acquisition and transmission of sexually transmitted infections [5] [6].

Streptococcus pyogenes ( $S$. pyogenes) is a gram-positive bacterium that infects the tonsils and pharynx. It is responsible for rheumatic fever, glomerulonephritis, and streptococcal toxic shock-like syndrome [7]. Although the pathogenesis of $S$. pyogenes has not been unclear, many virulent proteins are considered to be causative factors.

Although group B streptococci have been described as common members of the vaginal flora, vaginal colonization with $S$. pyogenes has been reported infrequently. Because of recent concern over the increasing incidence of severe, invasive $S$. pyogenes infections including vaginosis-induced streptococcal toxic shock-like syndrome [8], questions have been raised regarding the value of vaginitis for $S$. pyogenes [9].

The present study was conducted to find out the recent clinical characteristics of $S$. pyogenes isolates from vaginal discharge at general hospital in the central of Japan.

\section{Materials and Methods}

\subsection{Strains and Clinical Data}

A total of $61 S$. pyogenes was obtained from vaginal discharge at Daido Hospital between 2009 and 2017. Daido Hospital is a 404-bed private general hospital in the central region of Japan. We used medical records appended to clinical species for the analysis of clinical feature at Daido Hospital. We considered several isolates from the same region of the same patient as one isolate per one patient for the analysis in this study. All streptococcus isolates were identified by standard conventional biochemical methods or the VITEK2 system (bioMérieux, 
Durham NC, USA). Our experimental design was approved by the ethics committee at Daido hospital.

\subsection{Antimicrobial Susceptibility Analysis}

S. pyogenes isolates were examined for 10 antibiotic susceptibilities as follows AMPC; amoxicillin, CTM; cefotiam, CAZ; ceftazidime, CTRX; ceftriaxone, FOM; fosfomycin, MEPM; meropenem, CAM; clarithromycin, CLDM; clindamycin, MINO; minocycline, CPFX; ciprofloxacin. Minimal inhibitory concentrations (MICs) were determined at clinical laboratory in Daido Hospital using broth microdilution methodology with the VITEK2 system. Evaluation of susceptibilities was calculated based on Clinical Laboratory Standard Institute (CLSI) breakpoint [10].

\subsection{Statistical Analysis of the Data}

We conducted the statistical analysis with the chi-squared test or Fisher's exact test when appropriate. Differences were considered significant when $p$ was $<$ 0.05 .

\section{Results}

First of all, we evaluated that all $\mathcal{S}$. pyogenes isolates were beta hemolysis in this study. Next, we compared the differences among age. Furthermore we compared the differences between under and not less than 13 years age group in this study.

Figure 1 demonstrated the comparative analysis of $S$. pyogenes among age. Sixty-one $S$. pyogenes were isolated from vaginal discharge. The age incidence among 0 - 10 years age group was $14(23 \%)$, among $11-20$ years age group, 1 (1.6\%), among 21 - 30 years age group, 7 (11.5\%), among 31 - 40 years age group, 13 (21.3\%), among 41 - 50 years age group, 5 (8.2\%), among 51 - 60 years age group, 8 (13.1\%), among $61-70$ years age group, $11(18 \%)$, among $71-80$ years age group, $1(1.6 \%)$, and among 81 - 90 years age group, 1 (1.6\%). Next, we investigated the relationship between age distribution and ciprofloxacin susceptibility of $S$. pyogenes. The numbers of ciprofloxacin no-susceptible $S$. pyogenes from $0-10,31-40$, and $61-70$ years age group were higher than those from other groups (Figure 2). As we investigated the relationship between age distribution and clarithromycin susceptibility of $S$. pyogenes, the numbers of clarithromycin no-susceptible S.pyogenes from 0 - 10, 21 - 40, and 61 - 70 years age group were higher than those from other groups in Figure 3. Especially, all $S$. pyogenes from 21 - 30 years age group had clarithromycin no-susceptible ability $(p<0.05)$. Figure 4 showed that the numbers of clindamycin no-susceptible $S$. pyogenes from 0 - 10, and $21-40$ years age group were higher than those from other group with regard to the relationship between age distribution and clindamycin susceptibility of $S$. pyogenes. Figure 5 revealed that the numbers of minocycline no-susceptible $S$. pyogenes from 0 - 10, and 21 - 40 years age group were higher than those from other group. This antimicrobial pattern of 


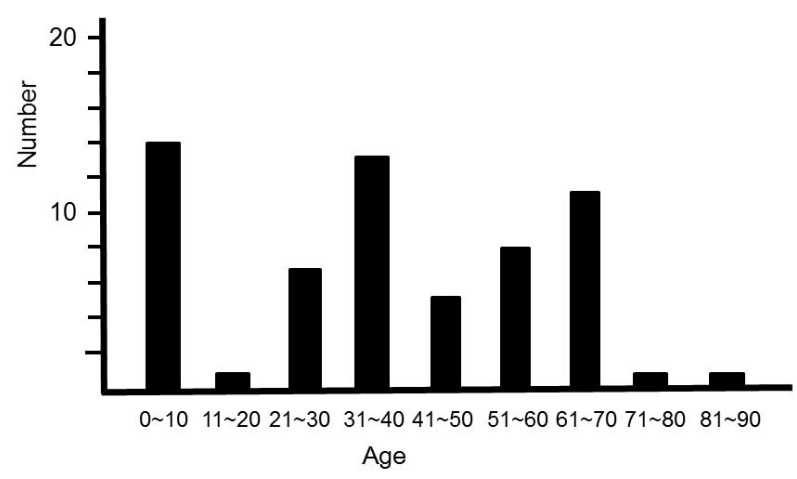

Figure 1. Age distribution of Streptococcus pyogenes isolates.

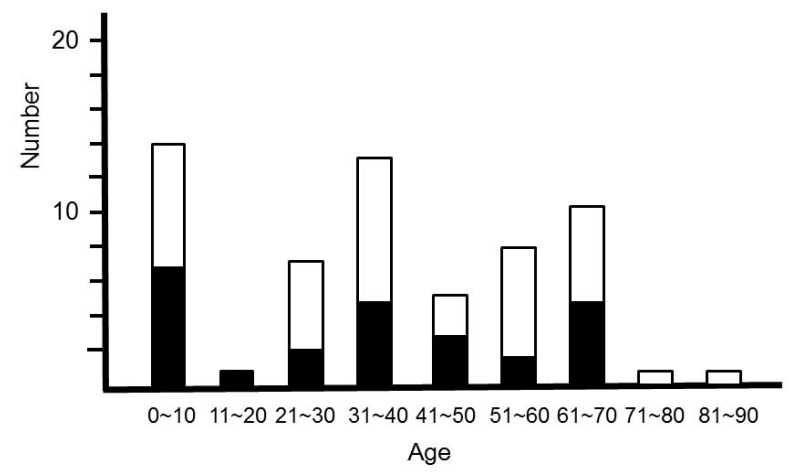

Figure 2. Ciprofoxacin susceptibility of Streptococcus pyogenes isolates. Black box: no-susceptible, white box: susceptible.

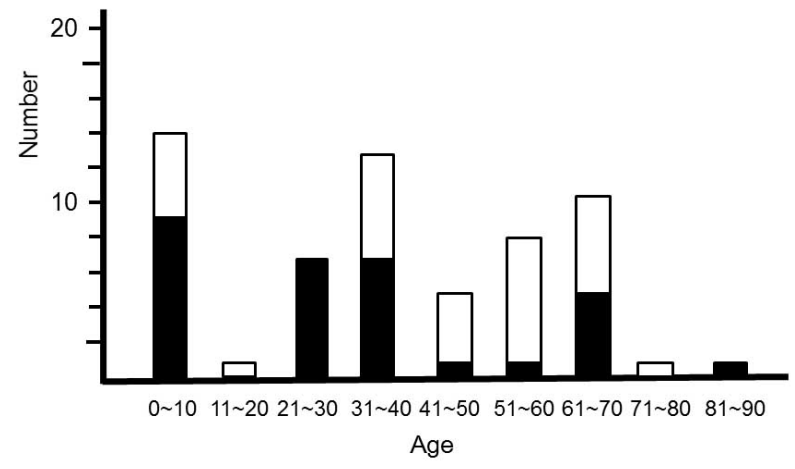

Figure 3. Clarithromycin susceptibility of Streptococcus pyogenes isolates. Black box: no-susceptible, white box: susceptible.

minocycline no-susceptible $S$. pyogenes was same as those of clarithromycin nosusceptible $S$. pyogenes. As other antibiotics were the most active antibiotics with $100 \%$ susceptible rates, we did not perform further analysis about these antibiotics. Furthermore, we analyzed the differences of four antimicrobial susceptible patterns (CPFX, CAM, CLDM, and MINO) both under and not less than 13 years age group. As the numbers of under and not less than 13 years age group were 15, and 46, respectively, the number of adult cases was higher than the number of child cases. The results of antimicrobial susceptible patterns of 


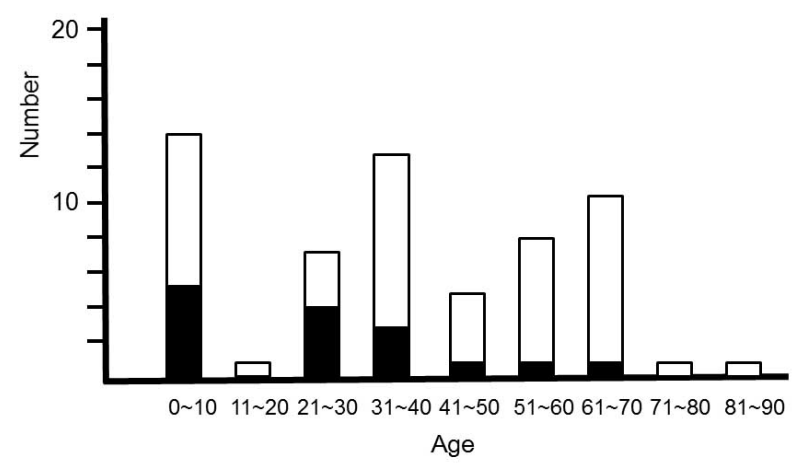

Figure 4. Clindamycin susceptibility of Streptococcus pyogenes isolates. Black box: no-susceptible, white box: susceptible.

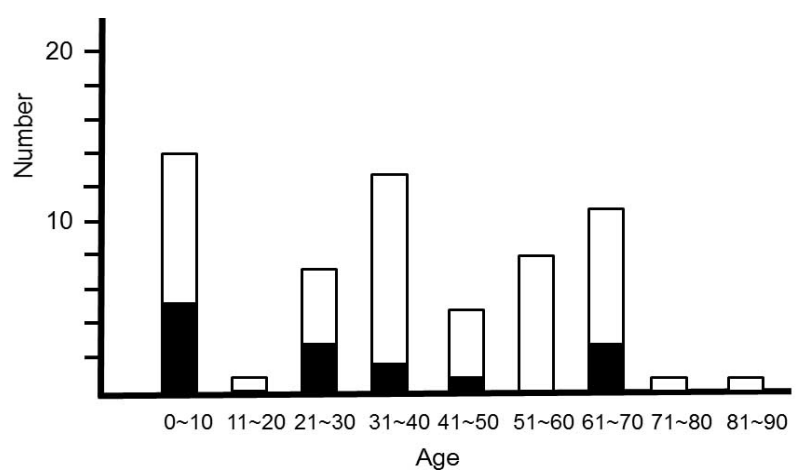

Figure 5. Minocycline susceptibility of Streptococcus pyogenes isolates. Black box: no-susceptible, white box: susceptible.

S.pyogenes isolates to four antibiotics tested in under 13 year's age group were shown in Figure 6. Clarithromycin no-susceptible rate was highest.

Both clindamycin and minocycline no-susceptible rate were same. Figure 7 demonstrated that clarithromycin no-susceptible rate was highest in not less than 13 years age group. Minocycline no-susceptible rate was lowest. There was no difference of antimicrobial susceptible trends between under and not less than 13 years age group.

\section{Discussion}

In this study, we described the characteristics of $S$. pyogenes isolates from vaginal discharge at general hospital in the central of Japan.

The present study revealed that the numbers of vaginal discharge-related $S$. pyogenes isolated from 0 - 10 years' children were most popular. However, the numbers of $S$. pyogenes isolated from 31 - 40 and 61 - 70 years' adult were also high.

In the analysis of antimicrobial susceptibility, the rate of ciprofloxacin and clindamycin no-susceptible $S$. pyogenes was similar in 0 - 10, 31 - 40, and 61 - 70 years' patients. However, especially, we found that clarithromycin was completely antimicrobial ineffective in 21 - 30 years' patients. Macrolide including clarithromycin is popular for treatment of several infectious diseases in Japan 


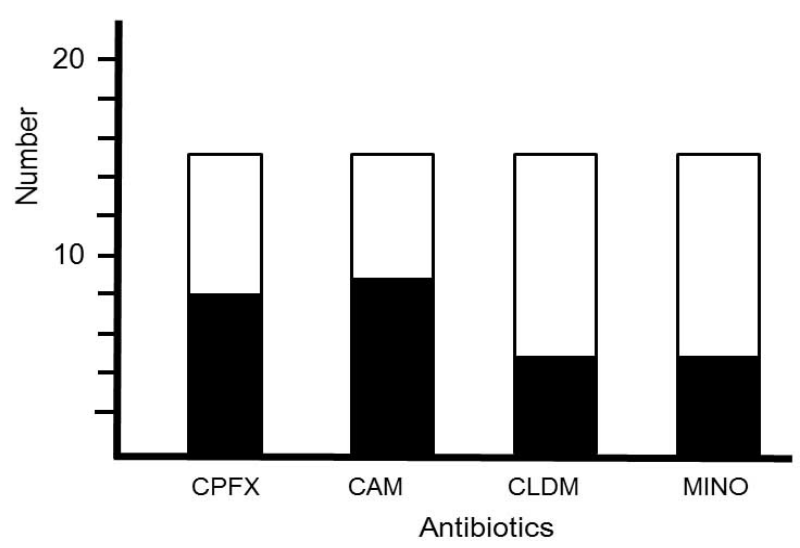

Figure 6. Four antimicrobial susceptibility of Streptococcus pyogenes isolates from under 13 years age group patients. Black box: no-susceptible, white box: susceptible. CPFX: ciprofloxacin, CAM: clarithromycin, CLDM: clindamycin, MINO: minocycline.

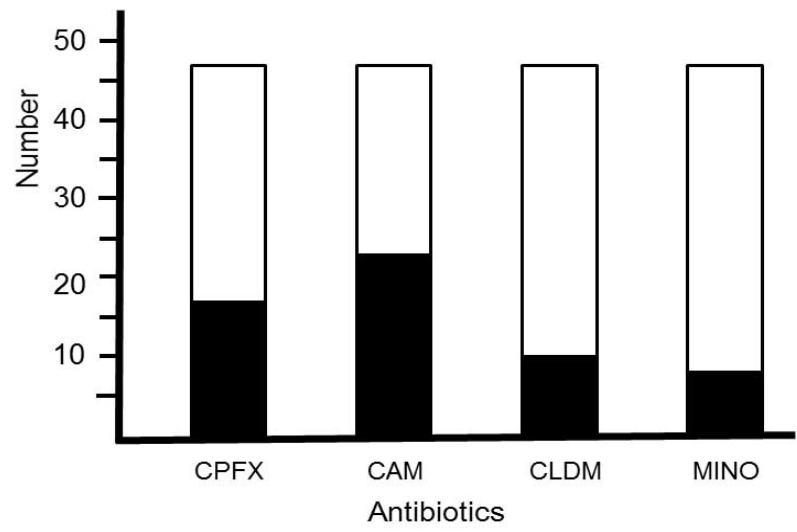

Figure 7. Four antimicrobial susceptibility of Streptococcus pyogenes isolates from not less than 13 years age group patients. Black box: no susceptible, white box: susceptible. CPFX: ciprofloxacin, CAM: clarithromycin, CLDM: clindamycin, MINO: minocycline.

[11]. Furthermore, low dose macrolide therapy is also popular for treatment of chronic disease [12] [13]. Our result suggested that inadequate usage of macrolide may increase the macrolide no-susceptible bacteria.

The comparative analysis between under and not less than 13 years age group demonstrated that ciprofloxacin and clarithromycin were less effective in $S$. pyogenes from about a half of under 13 years' patients. Even though $S$. pyogenes from a quarter of under 13 year's patients had clindamycin and minocycline no susceptibility, careful management must be taken in selecting antibacterial drugs for treatment of bacterial vaginosis. We found the numbers of bacteria from not less than 13 years' patients were about three times as same as those from under 13 years' patients. Streptococcal vaginosis was thought to be a disease that a child of 13 years old or less used to be [14] [15]. But recently we should change 
the perception that streptococcal vaginosis is also a disease that is an adult disease.

Further epidemiological analysis is needed for the clarification of several problems about vaginal discharge-associated $S$. pyogenes because the trend of infectious disease epidemiology is always changing drastically.

\section{Conclusion}

Our results showed that $S$. pyogenes-caused vaginosis was not children's illness, but an illness of adults, and that the prevalence of drug-resistant bacteria was also high. The results from our study strongly suggest the need for continuous epidemiological surveillance of $S$. pyogenes isolated from vaginal discharge.

\section{Acknowledgements}

We thank Mr. Masashi Ishihara and Ms. Miwako Fujimura for special encouragement. This study was supported by a grant-in-aid for research from the Nagoya City University, Japan.

\section{References}

[1] Eschenbach, D.A., Hillier, S., Critchlow, C., Stevens, C., DeRouen, T. and Holmes, K.K. (1988) Diagnosis and Clinical Manifestations of Bacterial Vaginosis. $A m J O b-$ stet Gynecol, 158, 819-828. https://doi.org/10.1016/0002-9378(88)90078-6

[2] Koumans, E.H., Sternberg, M., Bruce, C., McQuillan, G., Kendrick, J., Sutton, M., et al. (2007) The Prevalence of Bacterial Vaginosis in the United States, 2001-2004; Associations with Symptoms, Sexual Behaviors, and Reproductive Health. Sex Transm Dis, 34, 864-869. https://doi.org/10.1097/OLQ.0b013e318074e565

[3] Mitchell, C. and Marrazzo, J. (2014) Bacterial Vaginosis and the Cervicovaginal Immune Response. Am J Reprod Immunol, 71, 555-563.

https://doi.org/10.1111/aji.12264

[4] Muzny, C.A. and Schwebke, J.R. (2016) Pathogenesis of Bacterial Vaginosis: Discussion of Current Hypotheses. JInfect Dis, 214, S1-S5. https://doi.org/10.1093/infdis/jiw121

[5] Eschenbach, D.A. (1993) Bacterial Vaginosis and Anaerobes in Obstetric-Gynecologic Infection. Clin Infect Dis, 16, S282-S287.

https://doi.org/10.1093/clinids/16.Supplement_4.S282

[6] Hillier, S.L., Nugent, R.P., Eschenbach, D.A., Krohn, M.A., Gibbs, R.S., Martin, D.H., et al. (1995) Association between Bacterial Vaginosis and Preterm Delivery of a Low-Birth-Weight Infant. The Vaginal Infections and Prematurity Study Group. NEngl JMed, 333, 1737-1742. https://doi.org/10.1056/NEJM199512283332604

[7] Cunningham, M.W. (2000) Pathogenesis of Group A Streptococcal Infections. Clin Microbiol Rev, 13, 470-511. https://doi.org/10.1128/CMR.13.3.470-511.2000

[8] Hikone, M., Kobayashi, K., Washino, T., Ota, M., Sakamoto, N., Iwabuchi, S., et al. (2015) Streptococcal Toxic Shock Syndrome Secondary to Group A Streptococcus Vaginitis. JInfect Chemother, 12, 873-876. https://doi.org/10.1016/j.jiac.2015.07.011

[9] Mead, P.B. and Winn, W.C. (2000) Vaginal-Rectal Colonization with Group A Streptococci in Late Pregnancy. Infect Dis Obstet Gynecol, 8, 217-219. 
https://doi.org/10.1155/S1064744900000302

[10] Clinical and Laboratory Standards Institute (CLSI) (2014) Performance Standards for Antimicrobial Susceptibility Testing: 24th Informational Supplement, Clinical and Laboratory Standards Institute M100-S24, Wayne.

[11] Goto, H., Shimada, K., Ikemoto, H., Oguri, T., Study Group on Antimicrobial Susceptibility of Pathogens Isolated from Respiratory Infections (2009) Antimicrobial Susceptibility of Pathogens Isolated from More than 10,000 Patients with Infectious Respiratory Diseases: A 25-Year Longitudinal Study. J Infect Chemother, 15, 347-360. https://doi.org/10.1007/s10156-009-0719-3

[12] Cervin, A. and Wallwork, B. (2007) Macrolide Therapy of Chronic Rhinosinusitis. Rhinology, 45, 259-267.

[13] Kudoh, S. and Keicho, N. (2012) Diffuse Panbronchiolitis. Clin Chest Med, 33, 297-305. https://doi.org/10.1016/j.ccm.2012.02.005

[14] Stricker, T., Navratil, F. and Sennhauser, F.H. (2003) Vulvovaginitis in Prepubertal Girls. Arch Dis Child, 88, 324-326. https://doi.org/10.1136/adc.88.4.324

[15] Randjelovic, G., Otasevic, S., Mladenovic-Antic, S., Mladenovic, V., Radovanovic-Velickovic, R., Randjelovic, M., et al. (2017) Streptococcus pyogenes as the Cause of Vulvovaginitis and Balanitis in Children. Pediatr Int, 59, 432-437.

https://doi.org/10.1111/ped.13169 